\title{
Análise qualitativa do impacto do programa de treino metacognitivo e da cognição social em pessoas com Esquizofrenia
}

\author{
Nuno B F Rocha \\ Instituto Politécnico do Porto - Escola Superior de Tecnologia da Saúde \\ Cristina L Queirós \\ Universidade do Porto \\ Ana Ribeiro Bravo \\ Andreia $S$ A Silva \\ Terapeuta Ocupacional \\ António PS Marques \\ Instituto Politécnico do Porto - Escola Superior de Tecnologia da Saúde \\ Cláudia Oliveira \\ Terapeuta Ocupacional \\ Susana A Rocha \\ Instituto Superior de Contabilidade e Administração do Porto \\ Natacha G F Pereira \\ Terapeuta Ocupacional
}

\begin{abstract}
Resumo
Neste estudo procuramos determinar a satisfação e a percepção de mudanças específicas resultantes da participação num programa de treino metacognitivo e da cognição social (TMSC) para pessoas com Esquizofrenia. Participaram 21 participantes com diagnóstico de Esquizofrenia responderam a um inquérito por questionário de administração direta com questões abertas, realizado a meio e no final do TMCS. A partir das categorias (1) Satisfação com o programa, (2) Reestruturação sócio-cognitiva e (3) Mudanças na relação eu-outro, verificamos que o programa é relevante, tem potencial para introduzir mudanças na relação com os outros e parece otimizar a cognição social. Os aspectos negativos apontados estão relacionados com a duração do programa e das sessões, e com a linguagem utilizada.
\end{abstract}

Palavras-chave: esquizofrenia; cognição social; reabilitação psicossocial; metacognição.

\begin{abstract}
Qualitative analysis of the impact of the metacognitive and social cognition training in patients with Schizophrenia. This study aimed to understand the satisfaction and the perception of specific changes triggered by the participation in a metacognitive and social cognition training program (MSCT) for people with Schizophrenia. Participed 21 participants with a diagnosis of Schizophrenia answered a questionnaire of direct-response with open ended questions applied in the middle-term and at the end of the MSCT. Based on the categories (1) satisfaction with the program, (2) social cognitive restructuring and (3) Changes in the self-other relationship, we found that the program was considered to be relevant, had the potential to improve relationships with others and facilitated the enhancement of social cognition. The most important negative aspects mentioned were the duration of the program and sessions, and the specific language used.

Keywords: schizophrenia; social cognition; psychosocial rehabilitation; metacognition.
\end{abstract}

\section{Resumen}

Análisis cualitativo de los efectos del programa de entrenamiento metacognitivo y de la cognición social en personas con esquizofrenia. En este estudio hemos tratado de determinar la satisfacción y la percepción de los cambios específicos que resulten de la participación en un programa de entrenamiento metacognitivo y de la cognición social (TMSC) para las personas con esquizofrenia. 21 participantes con diagnóstico de esquizofrenia respondieron a un cuestionario de administración directa con preguntas abiertas, realizadas en el medio y al final de TMCS. A partir de las categorías (1) satisfacción con el programa, (2) reestructuración socio-cognitiva, y (3) cambios en la relación yo-otro, constatamos que el programa es relevante, tiene la 
posibilidad de introducir cambios en la relación con los demás y parece optimizar la cognición social. Los aspectos negativos se refieren a la duración del programa y de las sesiones, y la terminología utilizada.

Palabras clave: esquizofrenia; cognición social; rehabilitación psicosocial; metacognición.

$\mathrm{A}$ Esquizofrenia está associada a uma grave incapacidade de funcionamento e de participação social (American Psychiatric Association, 2000; Karagianis et al., 2009; Novick, Haro, Suarez, Vieta, \& Naber, 2009). Neste sentido, Green e colaboradores (2000) desenvolveram um trabalho de revisão sistemática da literatura e de meta-análise, que permitiu demonstrar que os déficits neurocognitivos (por exemplo, em relação à atenção, à memória e às funções executivas) podem contribuir para a incapacidade social. Designadamente, foram encontradas associações entre a ordenação de cartas (Wisconsin Card Sorting Test), a memória verbal secundária, a memória verbal imediata e a vigilância/atenção a pelo menos um tipo de resultados funcionais. Verificaram também que a predição da resposta funcional pela neurocognição pode variar entre os $20 \%$ e os $60 \%$. Green, Kern e Heaton (2004) mostraram ainda que as capacidades neurocognitivas avaliadas num momento podem predizer a funcionalidade a médio-longo termo. Contudo, apesar de ter tornado clara a existência de um impacto neurocognitivo na resposta funcional, há uma lacuna para explicar a restante variabilidade nos resultados funcionais (entre $40 \%$ e $80 \%$ ) que não é devida aos fatores neurocognitivos básicos.

Dados empíricos atuais revelam a presença de alterações severas na Esquizofrenia ao nível de um conjunto de funções especializadas no tratamento de informação social e afetiva e na representação dos estados mentais dos outros, que se designam por cognição social. Assim, para as pessoas com esta doença, torna-se extremamente complicado reconhecer as emoções (Chan, Li, Cheung, \& Gong, 2010; Edwards, Jackson, \& Pattison, 2002; Kohler \& Martin, 2006), processar pistas sociais e formular respostas a essas pistas (Green, Olivier, Crawley, Penn, \& Silverstein, 2005; Green et al., 2008; Leonhard \& Corrigan, 2001; Penn, Combs, \& Mohamed, 2001) e compreender e fazer a representação mental dos estados mentais dos outros (Bora, Yucel, \& Pantelis, 2009; Brune, 2005; Sprong, Schothorst, Vos, Hox, \& van Engeland, 2007; Tonelli \& Alvarez, 2009). Acrescentamos ainda a existência de vieses cognitivos que podem ter uma interferência considerável nos processos atributivos, na tomada de decisão e no processamento da informação social na Esquizofrenia. Destacamos o viés de personalização (Garety \& Freeman, 1999) e de intencionalização (Beck, Rector, Stolar, \& Grant, 2009), o viés de tirar conclusões precipitadas (Fine, Gardner, Craigie, \& Gold, 2007; Freeman, 2007; Garety, Hemsley, \& Wessely, 1991), e a convergência atribucional baseada em inferências monocausais (Moritz, Woodward, Burlon, Braus, \& Andresen, 2007; Randjbar, Veckenstedt, Vitzthum, Hottenrott, \& Moritz, 2011).

Estas alterações da cognição social parecem contribuir de forma única para a resposta funcional e para os sintomas clínicos, podendo para este efeito exceder os próprios processos neurocognitivos elementares (Couture, Penn, \& Roberts, 2006;
Fett et al., 2011; Garety \& Freeman, 1999). Nesse sentido, temos assinalado uma tendência para o desenvolvimento de programas específicos dirigidos ao treino da cognição social (Choi \& Kwon, 2006; Frommann, Streit, \& Wolwer, 2003; Horan et al., 2011; Kayser, Sarfati, Besche, \& Hardy-Baylé, 2006; Marsh et al., 2010; Mazza et al., 2010; Moritz, Woodward et al., 2011; Moritz \& Woodward, 2007; Roberts \& Penn, 2009; Roberts, Penn, Labate, Margolis, \& Sterne, 2010; Roncone et al., 2004; Silver, Goodman, Knoll, \& Isakov, 2004; Wolwer et al., 2005), fundamentados na hipótese que a optimização sócio-cognitiva pode ser acompanhada de benefícios funcionais e sintomatológicos. Trata-se de uma área muito promissora de intervenção, que, contudo, carece de maior investigação e sistematização para no futuro poder vir a ser considerada nos guidelines de tratamento da Esquizofrenia.

Dentre os vários programas existentes, salientamos pela sua relevância o Social Cognition and Interaction Training (SCIT) e o Metacognitive Training (MCT). Sendo dois programas com abordagens e enfoques diferentes, apresentam elementos complementares com grande relevância para a área das alterações da cognição social. O SCIT (Combs et al., 2007; Penn, Roberts, Combs, \& Sterne, 2007; Roberts \& Penn, 2009; Roberts et al., 2010) enfatiza os déficits da cognição social e os fatores que contribuem para as alterações atributivas que se registram na Esquizofrenia. É constituído por 18 sessões distribuídas por três fases (understanding emotions, figuring out situations e integration), onde se procura abordar os problemas de percepção emocional e de teoria da mente, a tomada de decisão precipitada e as atribuições sociais enviesadas. Já o MCT (Moritz, Woodward et al., 2011; Moritz, Veckenstedt, Randjbar, Vitzthum, \& Woodward, 2011; Moritz, Vitzthum, Randjbar, Veckenstedt, \& Woodward, 2010; Moritz \& Woodward, 2007) tem como objetivos principais ensinar o reconhecimento de distorções cognitivas e treinar formas alternativas de pensamento. Ao longo de oito módulos (neste programa cada sessão designa-se de módulo) os participantes aprendem a detectar e a atenuar armadilhas cognitivas automáticas, sendo evidenciadas estratégias de coping desadaptativas e as formas de as substituir por estratégias mais benéficas.

É precisamente neste sentido que adotamos neste trabalho o termo metacognição. Do ponto de vista etimológico, metacognição refere-se ao processo de pensar acerca do próprio pensamento (Flavell, 1979), que nos remete a uma das mais importantes capacidades humanas, que consiste em simultaneamente pensar e pensar acerca do pensamento. Se transportarmos esta ideia para o campo das relações sociais encontramos um conjunto infindável de situações em que há a necessidade de usar o conhecimento acerca dos estados mentais (do próprio e do outro) para adequar a resposta social. Por exemplo, está bem patente quando imaginamos o que outra 
pessoa está a pensar e a sentir, ou até mesmo quando (meta) representamos as suas intenções e crenças, sendo um conceito fundamental no estudo da teoria da mente (Goldman, 2006; Gopnik \& Wellman, 1994; Perner \& Howes, 1992). Do ponto de vista da intervenção, conceptualizamos o recurso à metacognição como forma de aumentar a consciência acerca das diferentes distorções cognitivas, de modo a facilitar a sua correção através do processo de refletir sobre o próprio pensamento.

Para este estudo, que se insere num projeto de investigação que visa o desenvolvimento e análise da eficácia de um programa de treino metacognitivo e da cognição social (TMCS) em pessoas com Esquizofrenia, estabelecemos como objetivo principal compreender o impacto da sua implementação com base na recolha e análise da perspectiva dos próprios participantes. Especificamente, pretendemos obter informações acerca da satisfação dos participantes com o programa, designadamente em relação à duração das sessões e do próprio programa, à pertinência dos temas abordados, à forma como decorreram as sessões e ao tipo de meios utilizados; e acerca da percepção de mudanças específicas resultantes do programa, nomeadamente no que diz respeito às modificações emocionais e sóciocognitivas, às alterações no relacionamento com os outros e à forma como a visão do mundo foi redefinida.

\section{Método}

\section{Procedimentos}

Inicialmente identificamos várias instituições que prestam cuidados no domínio da saúde mental a pessoas inseridas na comunidade e que se encontram vocacionadas para implementar programas de intervenção da natureza do TMCS. Após terem sido contactadas e de ter feito a apresentação da finalidade, objetivos e potenciais benefícios e inconvenientes do TMCS, obtivemos autorização formal de colaboração por parte de quatro Fóruns Sócio-Ocupacionais localizados em Portugal (ANARP e AFUA no distrito do Porto, Casa Ozanam no distrito de Aveiro e UNIR no distrito de Faro). Obtidas as autorizações por parte da direção das instituições que contactamos, foram identificados e encaminhados por parte da equipe técnica dos Fóruns os utentes que poderiam ser seguidos no estudo, de acordo com os critérios de seleção que estabelecemos inicialmente. Dos utentes sinalizados, participaram no programa TMCS aqueles que, sendo informados dos objetivos e procedimentos, decidiram colaborar voluntariamente, tendo-se recolhido o consentimento informado e garantido a confidencialidade e anonimato dos dados. Antes do início do programa, foi explicado a cada participante em que moldes se iria desenrolar o TMCS e as atividades que o compõem. Nesse momento foi dada a oportunidade para se esclarecerem as dúvidas existentes.

O TMCS consiste num programa de sessões de frequência bi-semanal, com a duração de 10 semanas, concebido para abordar de forma integrada as alterações da cognição social e os vieses sócio-cognitivos presentes na Esquizofrenia. Este programa é composto por uma componente de sessões de psicoeducação metacognitiva (Treino Metacognitivo) e por uma componente de sessões interativas da cognição social (Treino Interativo da Cognição Social), que decorrem de forma paralela. Cada sessão tem a duração de aproximadamente uma hora. $\mathrm{O}$ treino foi realizado em pequenos grupos, que variaram entre os três e os oito elementos por grupo.

A componente de treino metacognitivo baseia-se nos módulos descritos no manual desenvolvido por Moritz, Woodward e Rocha (2010). As sessões iniciam com a revisão do trabalho de casa da sessão anterior. Segue-se a introdução a um novo tema (relacionado com os vieses cognitivo), sendo ilustrada a falibilidade da cognição humana. São também realçados os extremos patológicos de cada viés cognitivo, explicando-se a forma como a exacerbação dos vieses (normais) de pensamento pode resultar em problemas da vida diária (ou inclusivamente culminar em delírios). As distorções cognitivas são elucidadas com recurso a exemplos e exercícios em grupos. No fim são elencados os pontos fundamentais de aprendizagem da sessão, com vista a uma melhor compreensão do alcance dos exercícios realizados no que diz respeito aos vieses sócio-cognitivos. $\mathrm{O}$ treino interativo de cognição social foca-se no processamento de pistas emocionais e sociais, na análise de situações sociais, na compreensão do discurso não literal, do logro e do humor, e na capacidade de tomar a perspectiva do outro. Para tal são utilizados exercícios de grupo, apresentações didáticas interativas, role-plays e trabalhos de casa. Os objetivos de cada componente do treino encontram-se resumidos na Tabela 1.

Os participantes responderam a um inquérito por questionário de administração direta com questões abertas, realizado a meio e no final do programa. As questões deste questionário encontramse Na Tabela 2.

Sobre as respostas às questões abertas fizemos incidir um tratamento qualitativo através de análise de conteúdo, com o objetivo de tornar manifestas e reais as categorias pré-definidas. As categorias de análise definidas para a nossa investigação foram as seguintes: (1) Satisfação com o programa, (2) Reestruturação sócio-cognitiva e (3) Mudanças na relação euoutro. Procuramos desta forma, aferir a satisfação e a percepção de efetividade do TMCS por parte dos participantes, bem como as modificações sócio-cognitivas e na vida diária que podem ser desencadeadas com este programa.

\section{Participantes}

Os dados deste estudo foram obtidos com base nos relatos de 21 participantes com Esquizofrenia (de acordo com os critérios do DSM-IV) que participaram durante 10 semanas no TMCS. Para a seleção dos participantes, definimos como critérios de exclusão a evidência de existência de doença neurológica (e.g., epilepsia, acidente vascular cerebral), a presença de um nível severo de deterioração cognitiva (ou mesmo deficiência mental) e a presença de perturbação de dependência de substâncias. Excluímos também todos os sujeitos que não se encontrassem compensados do ponto de vista psicopatológico. Incluímos apenas os doentes que tivessem idades compreendidas entre os 20 e os 55 anos de idades. Na tabela 3 apresentamos algumas características sócio-demográficas dos participantes deste estudo. 
Tabela 1

Objetivos dos Componentes do Treino Metacognitivo e da Cognição Social.

\begin{tabular}{|c|c|}
\hline \multirow{15}{*}{ Sessões metacognitivas } & Diferenciar atribuições externas, internas e circunstanciais. \\
\hline & $\begin{array}{l}\text { Identificar as vantagens e desvantagens relativas do estilo de atribuição depressivo e do viés em proveito } \\
\text { próprio. }\end{array}$ \\
\hline & $\begin{array}{l}\text { Sensibilizar para os riscos de se ignorarem os factos que são contrários às crenças pessoais ou de ativamente se } \\
\text { procurarem os que as confirmam. }\end{array}$ \\
\hline & Compreender que um incidente pode resultar de vários fatores. \\
\hline & Reconhecer a importância de se evitar tirar conclusões baseadas apenas nas primeiras impressões. \\
\hline & $\begin{array}{l}\text { Compreender que as situações podem mudar ao longo do tempo e que a evidência adicional não deve ser } \\
\text { ignorada. }\end{array}$ \\
\hline & $\begin{array}{l}\text { Adoptar um estilo de pensamento flexível que permita a revisão das crenças e a aceitação da evidência } \\
\text { contraditória. }\end{array}$ \\
\hline & Adotar critérios racionais nas tomadas de decisão. \\
\hline & $\begin{array}{l}\text { Considerar as informações contextuais, ao invés dos detalhes particulares e irrelevantes, para compreender os } \\
\text { estados mentais dos outros. }\end{array}$ \\
\hline & Diferenciar entre a informação conhecida pelo próprio e a informação disponível aos outros. \\
\hline & $\begin{array}{l}\text { Aceitar que em muitas situações da vida não se podem dar explicações definitivas sobre os assuntos, devendo- } \\
\text { se esperar pela obtenção de mais informações antes de se tirarem conclusões definitivas. }\end{array}$ \\
\hline & Compreender a falibilidade da memória humana: a memória é construtiva. \\
\hline & $\begin{array}{l}\text { Incentivar a recolha de informações adicionais quando não se tem uma recordação clara de determinado } \\
\text { assunto, particularmente em situações interpessoais significativas. }\end{array}$ \\
\hline & $\begin{array}{l}\text { Reconhecer estilos de pensamento disfuncionais que podem contribuir para a formação e manutenção de uma } \\
\text { baixa autoestima. }\end{array}$ \\
\hline & $\begin{array}{l}\text { Reforçar o uso de estratégias que melhorem a autoestima e que se oponham aos esquemas cognitivos } \\
\text { negativos. }\end{array}$ \\
\hline \multirow{10}{*}{$\begin{array}{l}\text { Treino interativo da } \\
\text { cognição social }\end{array}$} & Identificar e definir as emoções básicas (alegria, tristeza, zanga, medo, surpresa, nojo e vergonha). \\
\hline & Melhorar a capacidade de reconhecer as emoções básicas a partir de expressões faciais. \\
\hline & Compreender a causalidade emocional em situações sociais. \\
\hline & Identificar estratégias adaptativas para ultrapassar as emoções negativas e para manter as emoções positivas. \\
\hline & Reconhecer a diferença entre factos e interpretações em situações sociais. \\
\hline & Reconhecer que os outros detêm informações, sentimentos e pensamentos diferentes dos do próprio. \\
\hline & $\begin{array}{l}\text { Compreender que nas relações humanas as intenções nem sempre são explicitamente reveladas, não havendo } \\
\text { necessariamente uma má intenção subjacente. }\end{array}$ \\
\hline & $\begin{array}{l}\text { Compreender a necessidade de não se assumir literalmente tudo o que é comunicado e de distinguir este tipo } \\
\text { de comunicação nas situações sociais da vida diária. }\end{array}$ \\
\hline & Melhorar a capacidade de tomar a perspectiva do outro. \\
\hline & Implementar estratégias de resolução de problemas sociais em situações do dia-a-dia. \\
\hline
\end{tabular}

Tabela 2

Roteiro de Perguntas do Questionário Aplicado ao Participantes do Programa.

1. O Programa Metacognitivo e da Cognição Social está a corresponder às suas expectativas? (no final do programa: ...correspondeu às suas expectativas?)

2. Quais os temas que mais o estão a marcar? (no final do programa: ...que mais o marcaram?)

3. Acha o número de aulas suficiente? (no final do programa: Achou...).

4. Qual está a ser para si a utilidade deste Programa no seu dia-a-dia?

(no final do programa: Qual foi para si...).

5. Acha que o Programa está a contribuir para compreender melhor as emoções dos outros?

(no final do programa: Acha que o Programa contribuiu para...).

6. Acha que o Programa está a ajudá-lo a pensar melhor sobre as situações sociais?

(no final do programa: Acha que o programa ajudou-o a pensar...).

7. Mudou alguma coisa acerca da sua forma de pensar ao longo deste programa?

8. Indique os três aspectos mais positivos do programa.

9. Indique três aspectos negativos do programa

10. Há algum tema que gostaria de ver abordado e que não tenha sido? 
Tabela 3

Características Sócio-Demográficas dos Participantes. DPDesvio padrão; $M$-Média; M/F - Masculino/Feminino.

\begin{tabular}{|c|c|}
\hline & $\operatorname{TMCS}(n=21)$ \\
\hline Gênero (M/F) & $17 / 4$ \\
\hline Idade $\mathrm{M}(D P)$ & $39,00(8,83)$ \\
\hline \multicolumn{2}{|l|}{ Estado Civil } \\
\hline Casado & 2 \\
\hline Solteiro & 16 \\
\hline Divorciado/separado & 2 \\
\hline Viúvo & 1 \\
\hline \multicolumn{2}{|l|}{ Escolaridade } \\
\hline Inferior ao $9^{\underline{0}}$ ano & 7 \\
\hline $9^{-}-12^{-0}$ ano & 11 \\
\hline Ensino Superior & 3 \\
\hline \multicolumn{2}{|l|}{ Habitação } \\
\hline Própria & 5 \\
\hline Família de origem & 14 \\
\hline Residência & 2 \\
\hline \multicolumn{2}{|l|}{ Situação profissional } \\
\hline Desempregado & 8 \\
\hline Pensão social & 10 \\
\hline Reformado & 3 \\
\hline
\end{tabular}

\section{Resultados e análise interpretativa}

\section{Categoria satisfação com o programa}

Em primeiro lugar, sublinhamos que as respostas à questão 1 do questionário foram quase sempre positivas, tendo a maioria $(90,48 \%)$ dos participantes referido que o programa estava a superar as suas expectativas. Em geral, os participantes referiram estar satisfeitos com os conteúdos das sessões, conforme podemos ilustrar com afirmações como: “As sessões estão a ser positivas e estou a gostar"; “Útil, pois fala de temas que eu vivia mas não podia falar"; "Vão ao centro da questão, abordam temas importantes"; "É um programa interessante, compreensível"; "Muito útil, aprendemos coisas que raramente se aprende no Fórum e na vida quotidiana"; "Programa extremamente interessante, inteligente e criativo".

Não só os temas foram considerados relevantes, como a dinamização e organização das sessões parecem ter contribuído para uma boa participação, de acordo com expressões como: "Boa impressão"; "Têm sido dinâmicas e têm proporcionado interação"; "Sessões dinâmicas"; "Posso participar e responder a questões"; "Está a superar (as minhas expectativas) porque está a ser muito interativo, sessões práticas com interações entre os utentes"; "Cômico, alegre, importante e interessante".

A maior parte dos participantes $(71,43 \%)$ referiu que o número de sessões foi suficiente, apesar de termos recolhidos comentários que "talvez não" fossem suficientes ou que "não" foram suficientes, tendo mesmo havido um participante que indicou "não me importava de ter mais". Os temas mais marcantes relacionaram-se com a temática das "emoções", tendo os participantes pormenorizado que os marcaram o(s) ou a(s): (1)
Identificação das emoções pela expressão facial; (2) Sessões das emoções com as partes informatizadas; (3) Entender os sinais do rosto até para descobrir as emoções; (4) Tema das emoções das outras pessoas, conhecê-las melhor através dos gestos e das expressões faciais; (5) Antecipar sem precipitar a avaliação das emoções dos outros.

Outros temas frequentemente referidos foram a "empatia", a "memória", "saltar para conclusões", as "atribuições" e "humor e autoestima".

Não obstante a percepção de utilidade e de satisfação com o programa, alguns participantes $(28,57 \%)$ referiram ter sentido alguma fadiga durante as sessões, com comentários como "Sessões muito longas"; "Cansativas"; "Fico cansado"; "Semana intensiva"; "Torna-se cansativo, embora nos faça bem"; ou ainda "Horário (manhã, muito cedo), junção com outras atividades". Manteve-se, no entanto, a percepção da relevância do programa, com um participante a afirmar que "Tem sido muito diferente das atividades que faço aqui no Fórum. Estou a gostar, não tem sido difícil, mas custa-me muito estar na sessão o tempo todo".

Alguns participantes (33,33\%) manifestaram dificuldades a compreender os assuntos tratados, dada a sua exigência e complexidade, como podemos depreender dos comentários "Temas de difícil compreensão, sessões envolvem muita informação"; "A linguagem e compreensão dos slides era difícil, tive dificuldade"; "Linguagem difícil, dificuldade em compreender" e "O volume de informação é muito elevado". Por vezes a dificuldade situava-se na forma como os assuntos tocavam em aspectos estruturais na vida das pessoas, como um participante que referiu que "Partilhar as situações pessoais é difícil (fico a pensar), muito intensivo" e outro que indicou que "Muitas situações em que não consegui eliminar os juízos precipitados". Em todo o caso, para ilustrar a dificuldade de conciliar os níveis de complexidade a todos os participantes, houve um que indicou que "São aulas de pouca duração, são abordados um pouco superficialmente".

Quanto aos elementos facilitadores da aprendizagem, os participantes salientaram as tarefas de consolidação e generalização das aprendizagens, a estrutura operacional das sessões, a própria dinâmica de grupo, os meios audiovisuais e a vertente prática dos temas. Assim, algumas das expressões usadas foram: "Os trabalhos de casa ajudam a uma melhor aprendizagem, boa comunicação" (percebem-se perfeitamente todos os assuntos); "A matéria é agradável e do meu interesse"; "Sim, principalmente os TPC porque envolviam situações pessoais"; "Utilização de meios audiovisuais que ajudam na interiorização"; "Figuras e representar as emoções"; "Foi bom, pude colocar questões às outras pessoas e isso ajudou-me"; "Projeção de imagens, tempo de duração das sessões, grupo interessante"; "A visualização das imagens, a definição de emoções, conhecer realidades sociais"; "Sistema de dar a aula, interesse em nos fornecer informação"; "Aspectos cognitivos e práticos".

Resumindo, verificamos que os temas das sessões do TMCS foram em geral considerados relevantes. Para tal, contribuiu a forma como as sessões foram dinamizadas e os meios utilizados para a sua realização. Destaca-se a importância das atividades de generalização e de consolidação das aprendizagens. Retemos 
as informações relativas à presença de elementos que podem interferir com o treino, sobretudo relacionados com a linguagem empregue e com o volume de informação, que deverão ser equacionados no futuro.

\section{Restruturação sócio-cognitiva}

Relativamente a esta segunda categoria verificamos que a maior parte dos participantes $(80,95 \%)$ considerou que a sua forma de pensar mudou ao longo do programa, tendo esta opinião resultado essencialmente das respostas à questão 7 . Foram vastas as referências a uma sensação de mudança interna associadas a uma maior consciência metacognitiva, como podemos perceber com afirmações como: "Faz-me desenvolver mais a minha maneira de ser, de pensar, de agir, de falar com as pessoas"; "Ajuda a desenvolver capacidades"; "Maior atenção"; "Maior concentração"; "Maior ponderação"; "Melhor reconhecimento de sentimentos"; "Tornou-me mais ponderado"; "Ajudou-me a desmontar situações que por vezes eram dolorosas"; "Capacidade de raciocínio, crítica e memória"; "É importante, exercita o meu interior"; "Ajuda-me no dia-a-dia, ajuda a organizar as minhas ideias"; "Ajudou-me a pensar melhor"; "Ajudou a aperfeiçoar o meu pensamento"; "Aumentou o meu ângulo de visão"; "Para nos avaliarmos melhor perante determinadas situações, foi prático e eficaz".

Vários participantes $(66,67 \%)$ mencionaram o contributo do programa para o desenvolvimento de um estilo sócio-cognitivo mais prudente no que concerne à formulação de atribuições, como podemos perceber nas frases: "Mudou o fato de eu não tomar atitudes precipitadas"; "Já consigo pensar melhor nas decisões e não fazer julgamentos precipitados"; "Este programa ajudou-me a aceitar melhor a minha doença e perante uma situação damos várias hipóteses, aprendi a analisar melhor as expressões faciais e a não darmos como certas as primeiras impressões"; "Avaliar melhor [as] reações"; "Pensar que temos de pensar antes de algumas atitudes"; Ajudou a perceber que não me devo precipitar em tirar conclusões".

Apontamos anteriormente que a maioria dos participantes (80,95\%) indicou que Emoções tinha sido o tema que mais os tinha marcado. Parece certo que a relevância encontrada neste tema espelhou-se numa melhoria percebida da capacidade de reconhecimento emocional, como depreendemos quando alguns participantes observaram que: "Estou a compreender melhor a ver as emoções dos outros e a saber como estão e como se sentem e levar isso para lá para fora"; "Ajudou-me a usar o raciocínio e a ver as pessoas, aprender a avaliar as emoções dos outros"; "Agora tenho mais consciência das emoções dos outros, antes não ligava"; "Para ficarmos a conhecer melhor as expressões faciais no rosto das pessoas, conhecermo-nos melhor para perante uma determinada situação, pormos em análise e estudo várias hipóteses possíveis”; “Aprender a reconhecer, expressões corporais, julgamentos precipitados e crenças”.

Recolhemos também algumas referências ao fato de terem desenvolvido uma melhor autoestima, como depreendemos dos comentários "Mais autoestima e valor, saber como lidar com as minhas emoções", "Mudou. A saber, lidar com emoções dos outros, a ter autoestima e valor"; e "Sim, pelo fato de o programa ajudar a reduzir o estigma e o auto estigma".
Em suma, registamos que o TMCS produziu efeitos ao nível na capacidade dos participantes fazerem meta-representações sobre os seus próprios pensamentos. Estas modificações parecem ter tido implicações diretas na diminuição da propensão para se fazerem julgamentos precipitados ou pelo menos na reflexão crítica sobre os riscos desta forma de tomada de decisões. Sublinhamos ainda o desenvolvimento de uma autoestima mais positiva com o desenrolar do programa.

\section{Mudanças na relação eu-outro}

Grande parte das referências que obtivemos relativamente aos benefícios do TMCS prende-se com a redefinição da forma como as pessoas vêm os outros e o mundo que as rodeia. Em primeiro lugar, registamos uma melhor sensibilidade interpessoal, como podemos detectar nas expressões: "Estou a compreender melhor as pessoas"; "Entender melhor os outros"; "Conhecer melhor as pessoas"; "Sentia dificuldade em me pôr no lugar do outro e ainda sinto, apesar do que já aprendi. Mas já tenho mais consciência das emoções do outro"; "Ajuda-me a estar mais atento aos outros"; "Ainda tenho dificuldade em captar as emoções dos outros e prever os comportamento dos outros. Tem vindo a melhorar cada vez mais"; "Está a ajudar a tomar consciência dos outros e da minha maneira de estar com os outros".

Esta maior orientação para os outros parece ser acompanhada por uma melhor representação dos estados mentais do próprio, sobretudo quando lemos os comentários: "Já me compreendo melhor a mim e aos outros"; "Ajuda a compreender melhor os outros e a nós próprios, ajuda a sermos mais empáticos em relação aos outros, ajuda a ter uma melhor crítica e autocrítica"; "Reconheço melhor as minhas emoções e as dos outros"; "Compreendo melhor as interações entre as pessoas, empatia: já percebo melhor o que elas sentem. Ajudou a perceber melhor as minhas emoções".

Talvez o mais surpreendente seja o resultado deste processo de aproximação, que ao invés de ser ameaçador, pode converterse numa experiência positiva: "O que cada pessoa faz ou sente está a surpreender-me pela positiva" e "olho para as pessoas com outro otimismo".

Os participantes não só pareceram estar mais orientados para os outros, como também para o contexto envolvente, o que se torna evidente em afirmações como: "Tento extrair o contexto"; "Talvez para compreender melhor as situações do dia-a-dia"; "Aprendi a ver as emoções das pessoas e melhorei a parte cognitiva em relação às situações sociais"; "Pode ajudarme a não sentir-me tão culpada e a perceber melhor a situação. Vejo melhor as situações do dia-a-dia"; "há várias interpretações em diversas situações do quotidiano".

Outra área focada prende-se com a dimensão utilitária do programa, como depreendemos de expressões como: "Para aplicar no dia-a-dia nas situações que acontecem"; "Estou a tentar por em prática a ver as emoções das pessoas";

Está a ter utilidade para mim no meu dia-a-dia, no sentido de ter uma noção mais específica em relação às diversas e diferentes emoções... e não tirar conclusões precipitadas que me 
está a ajudar a pensar em várias soluções/conclusões para um determinado problema".

Foram feitas referências ao aumento do conforto social ("Ajuda a desinibir em público" e "Aprender a viver em sociedade"), à resolução de problemas ("Resolver situações de uma maneira eficaz e verdadeira", "Aprender a lidar com situações, emoções, não tomar atitudes precipitadas" e "Consegui ver as coisas de outra forma, aprendi outras coisas para lidar melhor com o meu dia-a-dia") e ao desenvolvimento de competências sociais ("Melhora as minhas competências sociais, relacionamento com os outros"). Contudo, foram feitas menções às dificuldades de utilização (generalização) das competências, como por exemplo, quando lemos os participantes afirmarem a impossibilidade de "Praticar aquilo que aprendemos. O tempo não foi suficiente" e de manifestarem que "É importante, embora tenha uma certa dificuldade em fazer aplicar aquilo que aprendi".

Outros não viram uma aplicação imediata dos temas tratados, admitindo, contudo, poderem vir a ser úteis no futuro, como quando referem que: "Não tem utilidade no dia-a-dia atualmente. Mas como os assuntos retratam situações que me podem vir a acontecer, pode ser útil no futuro para o meu relacionamento com os outros e comigo próprio"; "Não, mas no futuro pode vir a ser útil, pois fico mais informado e mais atento às pessoas";

Não está a ter impacto no dia-a-dia atualmente, mas penso que pode vir a ser útil em algumas situações de vida. Não ponho em prática, mas está a ser importante para a minha aprendizagem e no futuro aplicar em novas situações.

O principal objetivo dos programas de intervenção de raiz psicossocial prendem-se com a melhoria do funcionamento social. No que diz respeito às mudanças registradas na relação com os outros, e resumindo os dados que obtivemos, verificamos que o TMCS parece ter a potencialidade para sensibilizar os participantes para a necessidade de se compreender melhor os outros. Os resultados sugerem também que este aumento da sensibilidade interpessoal está relacionado com a capacidade de se fazerem representações dos estados mentais dos outros, que por sua vez parece ser uma consequência de uma maior capacidade de representação dos estados mentais do próprio. Sublinhamos o carácter utilitário do TMCS, designadamente no que diz respeito ao desenvolvimento de estratégias de resolução de problemas diários em situações sociais, não obstante alguns comentários que mostram as dificuldades que os doentes apresentam na transferência das aprendizagens para os seus contextos imediatos de vida diária.

\section{Considerações finais}

Nos últimos anos temos vindo a desenvolver uma proposta integrada de reabilitação e treino da cognição social para pessoas com incapacidades psiquiátricas do espectro da Esquizofrenia, que resultou na criação do programa treino metacognitivo e da cognição social. Neste estudo, procuramos analisar a percepção de pessoas com Esquizofrenia acerca da sua participação neste programa, incidindo na sua satisfação, nas reestruturações sócio- cognitivas desencadeadas e na mudança no relacionamento com os outros. Com estes dados pretendemos proceder aos ajustamentos necessários nos procedimentos do TMCS, de forma a conseguirmos chegar às necessidades e características da sua população alvo.

No que diz respeito aos aspectos positivos do programa, salientamos da análise qualitativa dos comentários dos participantes (1) a relevância dos temas, a sua dimensão utilitária, as tarefas de consolidação e generalização das aprendizagens e a estrutura operacional das sessões; (2) o potencial para optimizar a cognição social, para introduzir mudanças na forma de pensar e para reestruturar o processo de produção de atribuições; e (3) o impacto que pode ter na melhoria da sensibilidade interpessoal e na promoção da orientação para os outros e para o contexto envolvente. Por outro lado, identificamos alguns aspectos do TMCS que podem ser revistos, designadamente a duração do programa, que deve ser aumentada para facilitar a aquisição de competências e a generalização; a diminuição do tamanho de cada sessão, para evitar a fadiga; e a linguagem empregue, que deve ser mais próxima da realidade dos doentes, para melhorar a compreensão.

Na recente meta-análise de Kurtz e Richardson (2012), foram encontrados efeitos significativos da reabilitação da cognição social no reconhecimento emocional e na teoria da mente, o que se coaduna com os resultados que obtivemos. Podemos inferir que é possível melhorar nos doentes com Esquizofrenia as capacidades de meta-representação e de transposição para o ponto de vista dos outros. Muitos dos comentários dos participantes vão neste sentido. Não podemos ignorar que a teoria da mente envolve processos muito complexos de estimativa do estado mental dos outros, que são manifestamente difíceis de operacionalizar e transportar para sessões de intervenção (Horan et al., 2009). Os dados que recolhemos tornam evidente que existe esta possibilidade, com recurso a métodos metacognitivos.

Para além do estudo quase-experimental que levamos a cabo, no qual comparamos os resultados obtidos num grupo experimental que participou no TMCS com os de um grupo de doentes que se manteve em regime de tratamento habitual, em medidas de funcionalidade, sintomatologia e cognição social (o qual será brevemente publicado), propomos que em próximas investigações se proceda à aplicação deste programa de intervenção nas situações de primeiros surtos, ou até mesmo em grupos de alto risco para o desenvolvimento de psicose, sobretudo quando começam a surgir manifestações prodrómicas. Lembramos que são cada vez mais comuns os dados relativos à existência de alterações da cognição social nestas condições (Eack et al., 2010; Modinos, Renken, Shamay-Tsoory, Ormel, \& Aleman, 2010; Stanford, Messinger, Malaspina, \& Corcoran, 2011), o que poderá exigir uma resposta terapêutica precoce desta natureza.

Por outro lado, somos também da opinião de que a eficácia desta forma de intervir na cognição social deve beneficiar de um desenho experimental que envolva a randomização dos participantes em grupo experimental e de controlo. Somos, contudo, mais parcimoniosos nesta recomendação, por dois motivos. Em primeiro lugar, porque do ponto de vista ético deve ser muito bem pesada a decisão de não se envolver alguém 
numa intervenção notoriamente proveitosa, por um critério estatístico e não clínico. Em segundo lugar, porque um dos valores mais importantes da reabilitação psiquiátrica consiste no empowerment das pessoas com incapacidades psiquiátricas, que passa por entregar (ou devolver) o poder de decidir aos próprios indivíduos. Ora, sujeitá-los a um processo de aleatorização, no qual o próprio tem pouca escolha, pode ser contraproducente. Como em muitas outras situações de decisão clínica e de investigação, deverá imperar o bom senso.

$\mathrm{O}$ balanço final no que diz respeito à participação no TMCS parece ser bastante positivo, mesmo apesar das limitações inerentes a este tipo de intervenção. Como indicou um participante, e em jeito de resumo do que foi exposto, o programa parece ter ajudado a "Aprender a conhecer-me, a conhecer os outros e aprender a lidar com novas situações".

\section{Referências}

American Psychiatric Association. (2000). Diagnostic and Statistical Manual of Mental Disorders (4⿳⺈⿴囗十一 ed.). Washington: Autor.

Beck, A., Rector, N., Stolar, N., \& Grant, P. (2009). Schizophrenia: Cognitive Theory, Research and Therapy. Nova Iorque: The Guilford Press.

Bora, E., Yucel, M., \& Pantelis, C. (2009). Theory of Mind impairment in schizophrenia: Meta-analysis. Schizophrenia Research, 109(1-3), 1-9. doi: 10.1016/j.schres.2008.12.020

Brune, M. (2005). "Theory of Mind" in schizophrenia: A review of the literature. Schizophrenia Bulletin, 31(1), 21-42. doi: 10.1093/schbul/sbi002

Chan, R., Li, H., Cheung, E., \& Gong, Q. (2010). Impaired facial emotion perception in schizophrenia: A meta-analysis. Psychiatry Research, 178 , 381-390. doi: 10.1016/j.psychres.2009.03.035

Choi, K., \& Kwon, J. (2006). Social cognition enhancement training for schizophrenia: A preliminary randomized controlled trial. Community Mental Health Journal, 42(2), 177-187. doi: 10.1007/s10597-005-9023-6

Combs, D., Adams, S., Penn, D., Roberts, D., Tiegreen, J., \& Stem, P. (2007). Social cognition and interaction training (SCIT) for inpatients with schizophrenia spectrum disorders: Preliminary findings. Schizophrenia Research, 91(1-3), 112-116. doi:10.1016/j.schres.2006.12.010

Couture, S., Penn, D., \& Roberts, D. (2006). The functional significance of social cognition in Schizophrenia: A review. Schizophrenia Bulletin, 32 (suplemento 1), S44-S63. doi: 10.1093/schbul/sb1029

Eack, S. M., Mermon, D. E., Montrose, D. M., Miewald, J., Gur, R. E., Gur, R.C., ... Keshavan, M. S. (2010). Social cognition deficits among individuals at familial high risk for Schizophrenia. Schizophrenia Bulletin, 36(6), 10811088. doi: $10.1093 / \mathrm{schbul} / \mathrm{sbp} 026$

Edwards, J., Jackson, H., \& Pattison, P. (2002). Emotion recognition via facial expression and affective prosody in Schizophrenia: A methodological review. Clinical Psychology Review, 22, 789-832. doi: 10.1016/S02727358(02)00130-7

Fett, A., Viechtbauer, W., Dominguez, M. D., Penn, D. L., Os, J. v., \& Krabbendam, L. (2011). The relationship between neurocognition and social cognition with functional outcomes in Schizophrenia: A meta-analysis. Neuroscience and Biobehavioral Reviews, 35, 573-588. doi: 10.1016/j.neubiorev.2010.07.001

Fine, C., Gardner, M., Craigie, J., \& Gold, I. (2007). Hopping, skipping or jumping to conclusions? Clarifying the role of the JTC bias in delusions. Cognitive Neuropsychiatry, 12, 46-77. doi:10.1080/13546800600750597

Flavell, J. (1979). Metacognition and cognitive monitoring: A new area of cognitive-developmental inquiry. American Psychologist, 34, 906-911. doi:10.1037/0003-066X.34.10.906

Freeman, D. (2007). Suspicious minds: The psychology of persecutory delusions. Clinical Psychology Review, 27, 425-457. doi: 10.1016/j.cpr.2006.10.004
Frommann, N., Streit, M., \& Wolwer, W. (2003). Remediation of facial affect recognition impairments in patients with schizophrenia: A new training program. Psychiatry Research, 117(3), 281-284. doi:10.1016/S01651781(03)00039-8

Garety, P., \& Freeman, D. (1999). Cognitive approaches to delusions: A critical review of theories and evidence. British Journal of Clinical Psychology, 38, 113-154. doi:10.1348/014466599162700

Garety, P., Hemsley, D., \& Wessely, S. (1991). Reasoning in deluded schizophrenic and paranoid patients. Biases in performance on a probabilistic inference task. Journal of Nervous and Mental Disorders, 179, 194-201. doi:10.1097/00005053-199104000-00003

Goldman, A. (2006). Simulating minds: The philosophy, psychology, and neuroscience of mindreading. Oxford: Oxford University Press.

Gopnik, A., \& Wellman, H. (1994). The theory theory. In L. Hirschfeld \& S. Gelman (Orgs.), Mapping the mind: Domain specificity in cognition and culture (pp. 257-293). Nova Iorque: Cambridge University Press.

Green, M., Kern, R., \& Heaton, R. (2004). Longitudinal studies of cognition and functional outcome in schizophrenia: Implications for MATRICS. Schizophrenia Research, 72(1), 41-51. doi:10.1016/j.schres.2004.09.009

Green, M. F., Kern, R., Braff, D., \& Mintz, J. (2000). Neurocognitive deficits and functional outcome in schizophrenia: Are we measuring the "right stuff'? Schizophrenia Bulletin, 26(1), 119-136. doi:10.1093/oxfordjournals. schbul.a033430

Green, M. F., Olivier, B., Crawley, J., Penn, D., \& Silverstein, S. (2005). Social cognition in schizophrenia: Recommendations from the measurement and treatment research to improve cognition in schizophrenia new aproaches conference. Schizophrenia Bulletin, 31(4), 882-887. doi: 10.1093/schbul/ sbi049

Green, M. F., Penn, D. L., Bentall, R., Carpenter, W. T., Gaebel, W., Gur, R. C., .. Heinssen, R. (2008). Social cognition in schizophrenia: An NIMH workshop on definitions, assessment, and research opportunities. Schizophrenia Bulletin, 34(6), 1211-1220. doi: 10.1093/schbul/sbm145

Horan, W., Kern, R., Shokat-Fadai, K., Sergi, M., Wynn, J., \& Green, M. (2009). Social cognitive skills training in schizophrenia: An initial efficacy study of stabilized outpatients. Schizophrenia Research, 107(1), 47-54. doi: 10.1016/j. schres.2008.09.006

Horan, W., Kern, R., Tripp, C., Hellemann, G., Wynn, J., Bell, M., ...Green, M. F. (2011). Efficacy and specificity of social cognitive skills training for outpatients with psychotic disorders. Journal of Psychiatric Research, 45(8), 1113-1122. doi: 10.1016/j.jpsychires.2011.01.015

Karagianis, J., Novick, D., Pecenak, J., Haro, J., Dossenbach, M., Treuer, T., ... Lowry, A. J. (2009). Worldwide-schizophrenia outpatient health outcomes (W-SOHO): Baseline characteristics of pan-regional observational data from more than 17,000 patients. International Journal of Clinical Practice, 63(11), 1578-1588. doi: 10.1111/j.1742-1241.2009.02191.x

Kayser, N., Sarfati, Y., Besche, C., \& Hardy-Baylé, M. (2006). Elaboration of a rehabilitation method based on a pathogenetic hypothesis of "theory of mind" impairment in schizophrenia. Neuropsychological Rehabilitation, 16(1), 83-95. doi:10.1080/09602010443000236

Kohler, C., \& Martin, E. (2006). Emotional processing in schizophrenia. Cognitive Neuropsychiatry, 11(3), 250-271. doi:10.1080/13546800500188575

Kurtz, M., \& Richardson, C. (2012). Social cognitive training for schizophrenia: A meta-analytic investigation of controlled research. Schizophrenia Bulletin, 38(5), 1092-1104. doi: 10.1093/schbul/sbr036

Leonhard, C., \& Corrigan, P. (2001). Social perception in schizophrenia. In P. Corrigan \& D. Penn (Orgs.), Social cognition and schizophrenia (pp. 73-95). Washington, DC: American Psychological Association.

Marsh, P., Green, M., Russell, T. A., McGuire, J., Harris, A., \& Coltheart, M. (2010). Remediation of facial emotion recognition in schizophrenia: Functional predictors, generalizability, and durability. American Journal of Psychiatric Rehabilitation, 13(2), 143-170. doi:10.1080/15487761003757066 Mazza, M., Lucci, G., Pacitti, F., Pino, M., Mariano, M., Casacchia, M., 
\& Roncone, R.. (2010). Could schizophrenic subjects improve their social cognition abilities only with observation and imitation of social situations? Neuropsychological Rehabilitation, 20(5), 675-703. doi: 10.1080/09602011.2010.486284

Modinos, G., Renken, R., Shamay-Tsoory, S., Ormel, J., \& Aleman, A. (2010). Neurobiological correlates of theory of mind in psychosis proneness. Neuropsychologia, 48(13), 3715-3724. doi: 10.1016/j. neuropsychologia.2010.09.030

Moritz, S., \& Woodward, T. (2007). Metacognitive training in schizophrenia: From basic research to knowledge translation and intervention. Current Opinion in Psychiatry, 20, 619-625. doi:10.1097/YCO.0b013e3282f0b8ed

Moritz, S., Kerstan, A., Veckenstedt, R., Randjbar, S., Vitzthum, F., Schmidt, ... Woodward, T. S. (2011). Further evidence for the efficacy of a metacognitive group training in schizophrenia. Behaviour Research and Therapy, 49(3), 151-157. doi: 10.1016/j.brat.2010.11.010

Moritz, S., Veckenstedt, R., Randjbar, S., Vitzthum, F., \& Woodward, T. (2011). Antipsychotic treatment beyond antipsychotics: Metacognitive intervention for schizophrenia patients improves delusional symptoms. Psychological Medicine, 41(9):1823-32. doi: 10.1017/S0033291710002618

Moritz, S., Vitzthum, F., Randjbar, S., Veckenstedt, R., \& Woodward, T. (2010). Detecting and defusing cognitive traps: Metacognitive intervention in schizophrenia. Current Opinion in Psychiatry, 23, 561-569. doi: 10.1097/ YCO.0b013e32833d16a8

Moritz, S., Woodward, T., \& Rocha, N. (2010). Treino Metacognitivo Para Pacientes Com Esquizofrenia (TMC) - Manual (N. Rocha, C. Queirós, S. Aguiar, A. Marques \& S. Pereira, Trads.). Hamburg: VanHam Campus Press.

Moritz, S., Woodward, T., Burlon, M., Braus, D., \& Andresen, B. (2007). Attributional style in schizophrenia: Evidence for a decreased sense of selfcausation in currently paranoid patients. Cognitive Therapy and Research, 31(3), 371-383. doi: 10.1007/s10608-006-9070-5

Novick, D., Haro, J., Suarez, D., Vieta, E., \& Naber, D. (2009). Recovery in the outpatient setting: 36-month results from the schizophrenia outpatients health outcomes (SOHO) study. Schizophrenia Research, 108(1-3), 223-230. doi: 10.1016/j.schres.2008.11.007

Penn, D., Combs, D., \& Mohamed, S. (2001). Social cognition and social functioning in schizophrenia. In P. Corrigan \& D. Penn (Orgs.), Social Cognition and Schizophrenia (pp. 97-121). Washington, DC: American Psychological Association.

Penn, D., Roberts, D., Combs, D., \& Sterne, A. (2007). Best practices: The development of the social cognition and interaction training program for schizophrenia spectrum disorders. Psychiatric Services, 58(4), 449-451. doi: 10.1176/appi.ps.58.4.449

Perner, J., \& Howes, D. (1992). 'He thinks he knows': And more developmental evidence against the Simulation (Role Taking) Theory. Mind \& Language, 7, 72-86. doi: 10.1111/j.1468-0017.1992.tb00197.x

Randjbar, S., Veckenstedt, R., Vitzthum, F., Hottenrott, B., \& Moritz, S. (2011) Attributional biases in paranoid schizophrenia: Further evidence for a decreased sense of self-causation in paranoia. Psychosis, 3(1), 74-85. doi:10.1080/17522431003717675

Roberts, D., \& Penn, D. (2009). Social cognition and interaction training (SCIT) for outpatients with schizophrenia: A preliminary study. Psychiatry Research, 166(2-3), 141-147. doi: 10.1016/j.psychres.2008.02.007

Roberts, D., Penn, D., Labate, D., Margolis, S., \& Sterne, A. (2010). Transportability and feasibility of social cognition and interaction training (SCIT) in community settings. Behavioural and Cognitive Psychotherapy, 38(1), 35-47. doi: 10.1017/S1352465809990464

Roncone, R., Mazza, M., Frangou, I., Risio, A. D., Ussorio, D., Tozzini, C., \& Cassachia, M. (2004). Rehabilitation of Theory of Mind deficit in schizophrenia: A pilot study of metacognitive strategies in group treatment. Neuropsychological Rehabilitation, 14(4), 421-435. doi:10.1080/09602010343000291

Silver, H., Goodman, C., Knoll, G., \& Isakov, V. (2004). Brief emotion training improves recognition of facial emotions in chronic schizophrenia. A pilot study. Psychiatry Research, 128, 147-154. doi: 10.1016/j. psychres.2004.06.002

Sprong, M., Schothorst, P., Vos, E., Hox, J., \& van Engeland, H. (2007). Theory of Mind in schizophrenia: Meta-analysis. British Journal of Psychiatry, 191, 5-13. doi: 10.1192/bjp.bp.107.035899

Stanford, A., Messinger, J., Malaspina, D., \& Corcoran, C. (2011). Theory of Mind in patients at clinical high risk for psychosis. Schizophrenia Research, 131(1-3), 11-17. doi: 10.1016/j.schres.2011.06.005

Tonelli, H., \& Alvarez, C. E. (2009). Cognição social na esquizofrenia: Um enfoque em habilidades teoria da mente. Revista de Psiquiatria do Rio Grande do Sul, 31(suplemento 3), 0101-8108. doi: 10.1590/S010181082009000400005

Wolwer, W., Frommann, N., Halfmann, S., Piaszek, A., Streit, M., \& Gaebel, W. (2005). Remediation of impairments in facial affect recognition in schizophrenia: Efficacy and specificity of a new training program. Schizophrenia Research, 80(2-3), 295-303. doi: 10.1016/j.schres.2005.07.018 
Nuno B. F. Rocha, Doutor em Psicologia pela Faculdade de Psicologia e de Ciências da Educação da Universidade do Porto (FPCE-UP), é professor adjunto do Instituto Politécnico do Porto - Escola Superior de Tecnologia da Saúde (IPP-ESTS). Endereço para correspondência: IPP-ESTS, Rua Valente Perfeito, 322, 4400-330, Vila Nova de Gaia, Portugal. Tel.: 2220610 00. Email: nrocha@eu.ipp.pt

Cristina L. Queirós, Doutora em Psicologia pela Faculdade de Psicologia e de Ciências da Educação Universidade do Porto (FPCE-UP), é professora Auxiliar (FPCE-UP)

Email: cqueiros@fpce.up.pt

Ana Ribeiro Bravo, licenciada em terapia ocupacional pela Escola Superior de Tecnologia da Saúde do Porto (IPP-ESTS), é terapeuta ocupacional na Santa Casa da Misericórdia da Mealhada. Email: anaribeiro606@ gmail.com

Andreia S. A. Silva, licenciada em terapia ocupacional pela Escola Superior de Tecnologia da Saúde do Porto (IPP-ESTS), é terapeuta ocupacional na Associação Portuguesa de Pais e Amigos do Cidadão Deficiente Mental - Centro de Educação Especial de Castelo Branco (APPACDM). Email: to.asilva@gmail.com António P. S. Marques, Doutor em Psicologia pela Faculdade de Psicologia e de Ciências da Educação Universidade do Porto (FPCE-UP), é professor adjunto Instituto Politécnico do Porto - Escola Superior de Tecnologia da Saúde (IPP-ESTS).Email: ajmarques@estsp.ipp.pt

Cláudia Oliveira, licenciada em Terapia Ocupacional pela Escola Superior de Tecnologia da Saúde do Porto (IPP-ESTS), é terapeuta ocupacional na Associação de Familiares, Utentes e Amigos do Hospital Magalhães Lemos (AFUA). Email: claudiaoliveira.to@gmail.com

Susana A. Rocha, Mestrado em Psicologia pela Faculdade de Psicologia e de Ciências da Educação - Universidade do Porto (FPCE-UP), é assistente convidada no Instituto Superior de Contabilidade e Administração do Porto (IPP). Email: srocha@iscap.ipp.pt

Natacha G. F. Pereira, licenciada em Terapia Ocupacional pela Escola Superior de Tecnologia da Saúde do Porto (IPP-ESTS), é terapeuta ocupacional na Associação dos Doentes Mentais, Famílias e Amigos do Algarve (UNIR). Email: natachagranier@gmail.com 